\title{
ALARA (as low as reasonably achievable) CT 2011-executive summary
}

\author{
Beverley Newman • Michael J. Callahan
}

Received: 4 May 2011 / Accepted: 9 May 2011

(C) Springer-Verlag 2011

In August of 2001, under the guidance of Dr. Tom Slovis, the Society for Pediatric Radiology (SPR) organized the first of an ongoing series of multidisciplinary conferences to discuss current dose issues in pediatric imaging. This first meeting was dedicated to ALARA CT in children [1]. Since then there have been several additional, wellattended, SPR-sponsored ALARA conferences including computed/digital radiography [2], fluoroscopy and interventional radiology [3], emergency medicine [4] and oncology [5]. After a decade we have now come full circle back to $\mathrm{CT}$.

The sixth overall ALARA conference, and second ALARA CT conference, organized by Drs. Beverley Newman and Michael Callahan with the help of a superb group of advisers and the indispensable administrative assistance of Karen Schmitt, was held in Dallas, Texas, on March 5-6, 2011, sponsored by the SPR and ACR. Generous financial support for the conference was provided by GE Healthcare, Philips Healthcare, Siemens Healthcare, and Toshiba America Medical Systems. There were 15 course faculty members, including 12 radiologists, 2 medical physicists, and one pediatric emergency medicine physician. There were 130 attendees, including physicians, technologists, physicists and trainees. The conference consisted of six individual sessions, each with its own theme relevant to pediatric $\mathrm{CT}$ imaging and radiation dose.

B. Newman $(\bowtie)$

Department of Radiology, Lucile Packard Children's Hospital, Stanford University,

725 Welch Road,

Palo Alto, CA 94305, USA

e-mail: bevn@stanford.edu

M. J. Callahan

Department of Radiology, Children's Hospital Boston,

Harvard Medical School,

Boston, MA, USA
There were two lunch and dinner corporate educational sessions representing the four sponsoring vendors, who were each asked to address their approach to problems and solutions regarding dose reduction and ALARA CT in children.

The 2011 ALARA CT conference provided an international forum for the presentation of current, clinically relevant issues related to the practice of pediatric CT imaging. This supplement to the Pediatric Radiology journal contains peer-reviewed manuscripts from the material presented at the 2011 ALARA CT conference.

\section{Educational highlights of the 2011 ALARA CT conference}

Session 1: Progress in the last decade

Since the initial ALARA CT meeting nearly a decade ago, much has changed in terms of our general understanding of the biological effects of exposure to ionizing radiation $[6,7]$ and the impact of medically related radiation on the general population [8]. More important, there has been a culture change in how clinicians, radiologists, parents and patients understand the importance of the ALARA principle. As Dr. Slovis summed it up in his opening presentation, "Before 2000 pediatric radiologists were clueless and isolated" with regard to pediatric CT and dose. Since then numerous changes have occurred, outlined in a number of the presentations at the 2011 ALARA CT conference. These have included increased awareness, interest, knowledge, communication, culture and legislation, with pediatric radiologists spearheading efforts to justify and optimize CT imaging, use an evidence-based approach to $\mathrm{CT}$ versus other imaging alternatives and begin to take control of the technology rather than be controlled by it. 
Dr. Marilyn Goske highlighted the Image Gently campaign, guided by the Alliance for Radiation Safety in Pediatric Imaging, probably the highest-impact advance of the last decade in promoting pediatric radiation dose awareness and reduction. Founded in 2007 under the auspices of the SPR (Society for Pediatric Radiology), AAPM (American Association of Physicists in Medicine), ASRT (American Society of Radiologic Technologists) and ACR (American College of Radiology), Image Gently has developed an enormous global following and is currently affiliated with more than 60 national and international organizations. The Image Gently campaign has produced numerous educational modules that address dose reduction and appropriate imaging in children. The website (imagegently.org) contains reliable, peer-reviewed information for radiologists, technologists, physicists, clinicians and families, with subsections dedicated to pediatric $\mathrm{CT}$, interventional radiology, nuclear medicine and fluoroscopy. The alliance has acted as an important resource and advocate for children, has inspired the creation of the Image Wisely radiation campaign for adults and has promoted collaboration with imaging vendors as well as local, state, national and international agencies. Dr. Goske stressed that in spite of encouraging progress many challenges remain, particularly in light of the fact that $90 \%$ of pediatric CT emergency imaging is done in adult emergency departments.

Dr. Kimberly Applegate summarized the many public, governmental and medical educational resources that have become available and provide useful information regarding radiation dose. In addition to the Image Gently website, Dr. Applegate outlined the resources on the Health Physics Society website (radiationanswers.org); the International Atomic Energy Association's radiation protection of patients website (rpop.iaea.org); the ACR radiation safety site (acr.org) and the ACR-RSNA collaborative patient information website (radiologyinfo.org).

\section{Session 2: Physics and concepts}

Information presented addressed widely varying CT practices in both ordering and performance; practical methods of reducing CT dose and balancing dose and quality; understanding newer CT applications and technology in relation to dose and the inherent difficulties in accurately calculating dose and risk related to $\mathrm{CT}$ radiation exposure.

Dr. Keith Strauss presented the results of the AAPM taskforce 204 preliminary efforts to calculate accurate individual CT radiation exposure in children. He emphasized the deficiencies of the currently available scanner dose metrics, CTDI $_{\mathrm{vol}}$ and DLP, based on two sizes of acrylic CT phantoms $(16 \mathrm{~cm}$ and $32 \mathrm{~cm})$, with resultant markedly inaccurate estimations of radiation dose, usually underestimating patient dose, especially in small children. He described the AAPM's research-validated method of obtaining a size-corrected patient dose for chest and abdominal CT using the scanner CTDI and based on direct measurement of the AP and transverse dimensions of the individual patient either before (measured on scout) or after (measured on CT image) performance of the scan. Based on patient AP and lateral dimensions, a correction factor is calculated, which is multiplied by the $\mathrm{CTDI}_{\mathrm{vol}}$ to generate a size-corrected dose (SCD) expressed in mGy. While the exact utility of this metric is not yet defined, it is a major step forward in considering the specific dimensions of an individual patient and more accurately assessing that patient's true CT radiation exposure.

\section{Session 3: Clinical conundrums}

More clinically based topics addressed the issues and challenges of pediatric CT utilization in the emergency room setting and the difficulty of applying clinical decision models to children as well as possible appropriate considerations for use of MRI. Presentations by Dr. Sue Kaste on oncological surveillance and PET/CT in children highlighted the fact that few outcomes studies have evaluated surveillance imaging in detecting disease relapse after completion of therapy, and most of these studies "indicate little if any significant improvement in salvage rates between relapse detected by imaging or by clinical suspicions." Dr. Kaste emphasized the need for pediatric radiologists to be actively involved in designing, monitoring and analyzing sensitive, specific, innovative, evidence-based oncological protocols that are optimized for dose and clinical utility.

Session 4: Point/counterpoint (dose-related issues in CT)

The point/counterpoint session produced lively discussion of some of the more contentious CT imaging issues confronting pediatric radiologists. These included the when, who and how in performing sedation for imaging, when to gate for cardiac $\mathrm{CT}$ and the trade-offs of balancing dose reduction and diagnostic quality in both head and body imaging. To paraphrase Dr. Paul Guillerman, this should not be a race to the bottom; we should not be thinking only of Image Gently but rather Image IntelliGently.

Another topic that was thoroughly debated was the complexity of issues surrounding formal recording of $\mathrm{CT}$ radiation dose including the advantages and disadvantages, what should be recorded, by whom and where, who should have access to the information and how it could be used to improve standardization and quality control. 


\section{Session 5: Alternative imaging}

The talks took a change of pace looking at newer (often higher-dose) applications in $\mathrm{CT}$ and where they are appropriate for children as well as the advances in and applications of alternative non-ionizing imaging modalities such as US and MRI (advances include better quality and faster imaging related to higher field strength, improved coils, acceleration, navigation and advanced contrast agents).

International presentations by Drs. Karen Thomas and Paul Shrimpton in sessions 5 and 6 reflected on differences in pediatric CT utilization in other countries as well as differing clinical, legislative and radiation protection environments. We still have a long way to go and much to learn from experiences outside the U.S.

Session 6: Wrap-up-looking forward to future collaborative efforts

Speakers addressed some of the future challenges for CT such as current and likely future regulation and legislation as well as accountability, accreditation and certification. It was emphasized that national collaboration and ongoing educational efforts at all levels are needed to further improve understanding, knowledge and standardization of pediatric CT. Promising advances include: establishment of an ACR-sponsored national registry for pediatric CT to understand doses associated with current $\mathrm{CT}$ practice in children in order to provide a quality-improvement methodology for standardizing and optimizing practice and providing recommended dose limits (Quality improvement Registry for CT Scans in Children-QuiRCC); development of evidence-based materials for making appropriate imaging decisions including imaging-decision support as part of electronic order entry as well as improved methods of ongoing evaluation of educational and quality metrics in $\mathrm{CT}$.

\section{Conclusion}

While CT numbers and dose appear to be decreasing in pediatric hospitals [9], most pediatric CT imaging is performed outside these centers. There has been a promising start to collaborative educational and research efforts to provide guidance in the justification, optimization and standardization of pediatric CT imaging. Pediatric radiologists need to continue to work together collaborating on such projects as pediatric-specific CT dose and practice registries; education and standardization in pediatric CT justification and performance as well as quality control metrics. There is an ongoing need for collaborative evidence-based projects on specific pediatric disease entities; examples suggested by conference attendees included tumor surveillance imaging and $\mathrm{CT}$ imaging in cystic fibrosis and congenital heart disease. We also need to continue to develop and embrace robust radiation-free imaging modalities.

From the perspective of these editors one of the most important outcomes of this conference would be for credible, influential national organizations such as the ACR, Image Gently campaign, and the SPR and its official journal, Pediatric Radiology, to provide a sponsoring umbrella for the research and evaluation needed to establish, disseminate and promote widespread acceptance and adherence to standardized nomenclature and robust methods for calculating, recording and reporting CT dose in children. It is crucial to garner the support of our corporate imaging partners and other key interests for the methods adopted to become incorporated into automated and hopefully error-free electronic processes. Even if the initial choice is imperfect, standardization of dose metrics will promote greater understanding of CT dose in children and allow progress on the issues discussed and meaningful comparison of the many laudable projects that are already underway.

Acknowledgement Thanks to Pat Vario for indispensable administrative assistance in developing this supplement.

Disclaimer The supplement this article is part of is not sponsored by the industry. Dr. Newman and Dr. Callahan have no financial interest, investigational or off-label uses to disclose.

\section{References}

1. Slovis TL, Berdon WE (2002) The ALARA concept in pediatric CT intelligent dose reduction. Pediatr Radiol 32:217-317

2. Willis C (2004) Conference on the ALARA (as low as reasonably achievable) concept in pediatric CT intelligent dose reduction. Pediatr Radiol 34(Suppl 3):S159-S247

3. Kaste SC, Strauss K (2006) The ALARA (as low as reasonably achievable) concept in pediatric interventional and fluoroscopic imaging. Pediatr Radiol 36(Suppl 2):107-239

4. Frush D, Frush K (2008) The ALARA concept in pediatric imaging. Building bridges between radiology and emergency medicine: consensus conference on imaging safety and quality for children in the emergency setting. Pediatr Radiol 38(Suppl 4): S625-S734

5. Kaste SC (2009) The ALARA concept in pediatric imaging: oncology. Pediatr Radiol 39(Suppl 1):S1-S81

6. Brenner D, Elliston C, Hall E et al (2001) Estimated risks of radiation-induced fatal cancer from pediatric CT. AJR 176:289-296

7. Brenner DJ, Hall EJ (2007) Computed tomography_an increasing source of radiation exposure. N Engl J Med 357:2277-2284

8. Kase KR (2009) Report No. 160: ionizing radiation exposure of the population of the United States. National Council on Radiation Protection \& Measurements. Available via http:// www.ncrppublications.org/Reports/160. Accessed 11 May 2011

9. Townsend BA, Callahan MJ, Zurakowski D et al (2010) Has pediatric CT at children's hospitals reached its peak? AJR 194:1194-1196 